Rev. Elev. Méd, vét. Pays trop., 1977, 30 (3) . 303-308.

\title{
Essai sur l'incorporation de levure de brasserie séchée dans la ration de poulet d'engrais
}

\author{
par M. MAFWILA $\left(^{*}\right)$ et B. DIMBANI $\left(^{*}\right)$
}

\begin{abstract}
RÉSUMÉ
Quatre rations levurées à $1,2,3$ et 4 p. 100 ont été testées et comparées à une ration témoin. Les indices de consommation s'amélioraient avec le taux croissant de levure pour les rations qui en contenaient 1,2 et 3 p. 100, le meilleur indice étant réalisé avec le taux de 3 p. 100. Ce taux de 3 p. 100 a également assuré une meilleure évolution de poids tandis qu'une dépression de croissance fut observée au taux de 4 p. 100.
\end{abstract}

\section{INTRODUCTION}

Il est connu que les animaux domestiques peuvent utiliser avec profit les résidus industriels et que certains d'entre eux contiennent une teneur non négligeable en protéine. Ces sous-produits sont disponibles dans beaucoup d'industries locales des pays en développement.

Les brasseries du Zaïre n'accordent généralement aucune importance à leurs sous-produits : la drêche est habituellement jetée au fleuve bien que, dans certaines brasseries, elle trouve déjà des débouchés auprès des meuneries et d'éleveurs intéressés qui en sollicitent. Ceci ne constitue nullement une activité commerciale intense de la part des brasseries étant donné que ces approvisionnements sont faibles et irréguliers. Quant à la levure, aucune des brasseries visitées ne la valorise.

C'est dans le but de valoriser la levure de brasserie que nous l'avons retirée des cuves de fermentation d'une brasserie, lavons bien séchée et incorporée dans la ration de poulet d'engrais afin d'en étudier les effets.

(*) Institut Facultaire des Sciences Agronomiques, B. P. 28 Yangambi, Zäre.

(**) Institut Supérieur d'Etudes Agronomiques, B. P. 202 Kisangani, Zaïre.

\section{GENERALITES}

Dans les brasseries, une partie de levure peut être récupérée après filtration du moût. Séché, ce sous-produit peut être utilisé pour l'alimentation des animaux domestiques ; le tableau $n^{\circ} I$ nous informe sur sa valeur nutritive.

La levure de brasserie séchée est donc un sous-produit riche en protéine et contenant des quantités appréciables de vitamines du complexe B ; par ailleurs, ses acides aminés sont équilibrés. Elle se présente sous forme de paillettes plus ou moins granuleuses et de couleur jaune, elle se mélange facilement aux autres ingrédients de la ration.

La levure séchée peut être incorporée aux rations de volailles dans les proportions de 2 à 5 p. 100 mais cependant son caractère laxatif en limite le taux d'incorporation. EWING (3) rapportant les expériences d'autres auteurs chez la volaille, souligne l'influence positive de ce sous-produit sur la ponte, l'abaissement du taux de mortalité et l'efficacité alimentaire; néanmoins, aucune influence n'a pu être constatée sur la couleur du jaune d'œuf et d'autres qualités intrinsèques de l'œuf. 
TABL. $\mathrm{N}^{\circ}$ I-Composition de la levure de brasserie sêchêe.

\begin{tabular}{|c|c|c|c|}
\hline Matiêre brute & p. 100 & & $\begin{array}{l}\mathrm{p} \cdot 100 \\
\text { ou } \mathrm{mg} / \mathrm{kg}\end{array}$ \\
\hline Eau & 10,90 & $\begin{array}{l}\text { Total digestible nutrients } \\
\text { p. } 100\end{array}$ & 68,09 \\
\hline Matière sèche & 89,10 & Unitê fourragère & 0,88 \\
\hline Protéine brute & 48,29 & $\begin{array}{l}\text { Energie métabolisable } \\
\mathrm{kcal} / \mathrm{kg}\end{array}$ & 2794 \\
\hline Protêine digestible & 42,46 & Manganèse $(\mathrm{mg} / \mathrm{kg})$ & 2,40 \\
\hline Cellulose & 0,88 & Fer p. 100 & 0,0138 \\
\hline Matière grasse & 0,55 & Cuivre $(\mathrm{mg} / \mathrm{kg})$ & 15,10 \\
\hline Matière minêrale & $7-7,5$ & Cobalt $(\mathrm{mg} / \mathrm{kg})$ & 0,08 \\
\hline Calcium & 0,088 & Thiamine $(\mathrm{mg} / \mathrm{kg})$ & 43,00 \\
\hline Phosphore & 1,749 & Riboflavine (mg/kg) & 14,00 \\
\hline Extractif non azotê & 30,58 & Niacine (ng $/ \mathrm{kg})$ & 213,00 \\
\hline
\end{tabular}

(Sources : Ewing, 1951 ; Mongadin et Rivière, 1965)

WEGNER (3) utilisa des rations de poulets de chair contenant de la levure : levure + drêche de brasserie, levure + son de blé à concurrence de 5 p. 100 par variante. Chez les femelles, l'évolution de poids et la consommation étaient semblables dans les différents groupes mais on remarquait la tendance d'une amélioration de la croissance et de l'indice de consommation chez les rations levure + drêche et levure + son, tout en étant plus riches en fibre.

Chez les mâles, les rations précitées assuraient une meilleure croissance et un meilleur indice de consommation si bien que les combinaisons levure + drêche et levure + son ne portent pas atteinte aux performances des poulets de chair.

\section{MATERIEL ET METHODES}

\section{Milieu expérimental}

L'expérience a été menée à la ferme avicole Munama, propriété de la Société Générale d'Alimentation (S. G. A.), située à environ $3 \mathrm{~km}$ du centre de la ville de Lubumbashi (exElisabethville). Les données géographiques de cette ville sont les suivantes : $11^{\circ} 29^{\prime}$ latitude Sud, $27^{\circ} 28^{\prime}$ longitude Est et $1200 \mathrm{~m}$ d'altitude.

\section{Equipement}

Le poulailler ayant servi à l'essai fut au préalable désinfecté et il réunissait de bonnes conditions d'éclairage, d'aération, d'affouragement et d'abreuvement. Les poulets ont été élevés dans 2 batteries comprenant chacune 5 cages superposées. Les dispositifs de chauffage réglables n'ont été mis en marche que pendant les nuits des deux premières semaines.

\section{Animaux d'expérience et mesures pro- phylactiques}

Un total de 1400 broilers d'un jour non sexés et de souche Arbor Acres en provenance de Zambie a été nourri d'un aliment commercial approprié pendant une période pré-expérimentale de 2 semaines. Pour l'expérience qui dura 4 semaines, 250 poussins ont été prélevés et répartis au hasard en 10 lots $(5 \times 2$ répétions) de 25 individus dans les 2 batteries.

Des mesures prophylactiques ont été appliquées, notamment l'administration d'un antistress dans l'eau de boisson au cours des 4 premiers jours de vie, la vaccination contre la maladie de Newcastle le $5^{\circ}$ et le $21^{\circ}$ jour ainsi que l'utilisation d'un anticoccidien du $14^{*}$ au $16^{*}$ jour.

Après un mois, nous pouvions distinguer les mâles et les femelles et avons remarqué que l'effectif contenait plus de femelles que de mâles mais que, dans chaque groupe, le nombre de mâle était presque le même; ainsi le poids des mâles qui est généralement supérieur à celui des femelles ne risque pas d'influencer les résultats. 
TABL. $N^{\circ}$ II-Formules des rations en $\mathrm{p}, 100(1)$

\begin{tabular}{|l|c|c|c|c|c|}
\hline \multicolumn{1}{|c|}{ Ingrédients } & $\mathrm{R}_{t}$ & $\mathrm{R}_{1}$ & $\mathrm{R}_{2}$ & $\mathrm{R}_{3}$ & $\mathrm{R}_{4}$ \\
\hline Farıne de maís jaune & 43,45 & 43,45 & 43,45 & 43,45 & 43,45 \\
Tourteau d'arachide & 15,00 & 15,00 & 15,00 & 14,60 & 14,50 \\
Farine de viande & 16,00 & 16,00 & 15,50 & 15,50 & 15,30 \\
Farine de manioc & 22,70 & 21,70 & 21,20 & 20,60 & 19,90 \\
Levure de brasserie & 0,00 & 1,00 & 2,00 & 3,00 & 4,00 \\
séchée & 2,50 & 2,50 & 2,50 & 2,50 & 2,50 \\
Condiment minéra1 & 0,35 & 0,35 & 0,35 & 0,35 & 0,35 \\
et vitaminê & & & & \\
Sel de cuisine & & &
\end{tabular}

(1) $R_{t}=$ ration témoin; $R_{1}$ à $R_{4}=$ rations supp lémentées.

\section{Levure de brasserie}

Il faut noter que la levure qui a servi pour l'expérience avait été retirée des cuves de fermentation de la brasserie Simba à Lubumbashi. Elle a ensuite été séchée au soleil dans des bacs métalliques ondulés d'environ $1 \times 1,20 \mathrm{~m}$ pendant 3 à 4 jours.

Son analyse au laboratoire a donné les valeurs suivantes : 91 p. 100 de matière sèche, 43 p. 100 de protéine brute, 0,80 p. 100 de matières grasses brutes et 13 p. 100 de cendres brutes.

\section{Constitution des rations}

La levure de brasserie séchée a été ensuite incorporée dans 4 rations aux doses de 1,2 , 3 et 4 p. 100 respectivement dans les rations $R_{1}, R_{2}, R_{3}$ et $R_{4}$ comme le montre le tableau $\mathrm{n}^{\circ}$ II.

Le condiment minéral et vitaminé utilisé avait la composition suivante : $P 19,6$ p. 100 ; Ca 25,5 p. $100 ; \mathrm{Na} 2,4$ p. 100 ; Mg 1,2 p. 100 et 300000 UI Vit. A au kg; 50000 UI Vit. D3 au $\mathrm{kg} ; 100 \mathrm{mg}$ Vit. E au kg.

Les rations ont été calculées de manière à contenir une teneur analogue en protéine et à satisfaire les besoins en d'autres principes alimentaires. Le tableau $\mathrm{n}^{\circ}$ III nous donne la composition bromatologique des différentes rations.

TABL. N ${ }^{\circ}$ III-Composition bromatologique des rations.

\begin{tabular}{|l|c|c|c|c|c|}
\hline & $\mathrm{R}_{\mathrm{t}}$ & $\mathrm{R}_{1}$ & $\mathrm{R}_{2}$ & $\mathrm{R}_{3}$ & $\mathrm{R}_{4}$ \\
\hline Matière sèche & 90,00 & 90,00 & 88,57 & 90,00 & 92,85 \\
Protéine brute & & & & & \\
- calculée & 20,87 & 21,23 & 21,31 & 21,66 & 21,97 \\
- analysée & 19,37 & 20,00 & 20,31 & 20,63 & 20,63 \\
Matière grasse brute & 4,10 & 4,15 & 4,15 & 4,20 & 4,22 \\
Fibre brute & 2,71 & 2,69 & 2,65 & 2,63 & 2,61 \\
Cendre brute & 6,50 & 8,00 & 7,50 & 8,00 & 8,00 \\
Calcium & 2,08 & 2,08 & 2,04 & 2,04 & 2,03 \\
Phosphore & 1,43 & 1,44 & 1,43 & 1,44 & 1,45 \\
Rapport Ca/P & 1,46 & 1,46 & 1,42 & 1,42 & 1,40 \\
Energie mé tabolisab1e & 2871 & 287 & 2869 & 2867 & 2906 \\
kcal/kg & 138 & 135 & 135 & 132 & 132 \\
Rapport C/P & & & & & \\
\hline
\end{tabular}


TABL. $\mathrm{N}^{\circ}$ IV-Rẻsultats de $I^{\prime}$ expérience

\begin{tabular}{|l|c|c|c|c|c|}
\hline & $\mathrm{R}_{\mathrm{t}}$ & $\mathrm{R}_{\mathrm{I}}$ & $\mathrm{R}_{2}$ & $\mathrm{R}_{3}$ & $\mathrm{R}_{4}$ \\
\hline Poids total initial $(\mathrm{g})$ & 9369 & 9394 & 9410 & 9541 & 9714 \\
Poids moyen initial $(\mathrm{g})$ & 187,38 & 187,88 & 188,20 & 190,82 & 194,28 \\
Poids total final (g) & 24495 & 25650 & 27205 & 26620 & 25965 \\
Poids moyen rinal (g) & 489,90 & 513,00 & 544,10 & 572,40 & 519,30 \\
Gain de poids moyen (g) & 302,52 & 325,12 & 355,90 & 381,58 & 325,02 \\
Consommation moyenne $(\mathrm{g})$ & 972,00 & 1003,40 & 999,00 & 1026,00 & 952,00 \\
Indice cons onmation (+) & 3,21 & 3,09 & 2,81 & 2,72 & 2,93 \\
\hline
\end{tabular}

$(+)=\frac{\text { Cons ommation moyenne }}{\text { Gain de poids moyen }}$

\section{RESULTATS ET INTERPRETATION}

Le tableau $\mathbf{n}^{0}$ IV donne les différents résultats obtenus pour les 2 batteries et la figure 1 reproduit les courbes de croissance des sujets soumis aux différentes rations. Considérant l'évolution des courbes de croissance, nous

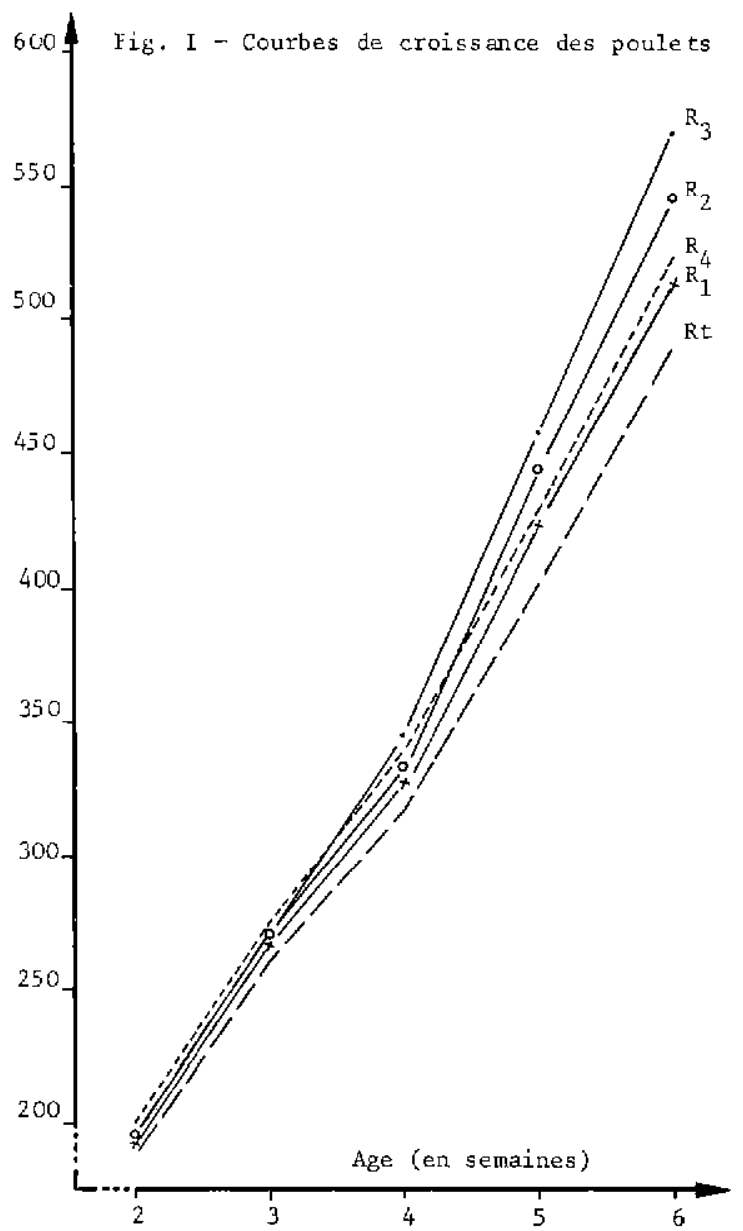

constatons que les rations se rangent dans l'ordre suivant : $\mathbf{R}_{3}, \mathbf{R}_{2}, \mathbf{R}_{4}, \mathbf{R}_{1}$ et $\mathbf{R}_{\mathbf{1}}$ allant ainsi de la meilleure à la moins bonne; cette tendance est très remarquable à partir de la $2^{*}$ moitié de l'expérience.

L'analyse de variance à deux causes contrôlées a révélé l'effet batterie et l'interaction batterie-ration au seuil de probabilité de 5 p. 100 alors que l'effet ration a joué au seuil de probabilité de 1 et 5 p. 100 . Cependant, la même analyse effectuée à partir des gains de poids hebdomadaires moyens et des indices de consommation ne décela aucune différence.

Poursuivant avec le DUNCAN-Test sur les poids finals moyens, les résultats suivants ont été obtenus :

Pas de différence $(P>5$ p. 100$)$ entre :

$$
R_{1}-R_{4}
$$

Différence légèrement significative $(\mathrm{P} \leqslant$ 5 p. 100) entre :

$$
\begin{aligned}
& \mathrm{R}_{\mathrm{t}}-\mathrm{R}_{1} ; \mathrm{R}_{4} \\
& \mathrm{R}_{1}-\mathrm{R}_{2} \\
& \mathrm{R}_{2}-\mathrm{R}_{3} ; \mathbf{R}_{4}
\end{aligned}
$$

Différence significative ( $\mathrm{P} \leqslant 1$ p. 100) entre :

$$
\begin{aligned}
& \mathbf{R}_{3}-\mathbf{R}_{\mathbf{t}} ; \mathbf{R}_{1} ; \mathbf{R}_{4} \\
& \mathbf{R}_{\mathbf{t}}-\mathbf{R}_{2}
\end{aligned}
$$

\section{DISCUSSION}

Les poids finals moyens obtenus dans cette expérience rangent la ration $\mathrm{R}_{3}$ contenant 3 p. 100 de levure en tête de toutes les autres rations. Elle est suivie respectivement par la ration $R_{2}$, la ration $R_{4}$, la ration $R_{1}$ et la ration témoin $\mathbf{R}_{\mathbf{t}}$. 
Le même ordre est suivi par les rations quant aux gains de poids moyens, sauf que l'ordre entre les rations $R_{1}$ et $R_{4}$ s'est inversé mais la différence entre elles n'est que de $0,10 \mathrm{~g}$.

Les poids moyens atteints dans cet essai oscillent entre 489,90 et $572,40 \mathrm{~g}(\varnothing 527,74 \mathrm{~g}$ ) et sont inférieurs au poids de $720-880 \mathrm{~g}$ que peuvent atteindre, selon BRION (1), les poulets de chair à 6 semaines. Cette différence pourrait s'expliquer par la souche utilisée et par plusieurs autres facteurs.

Toutefois, I'objectif de la ferme Munama qui est d'obtenir des poussins à croissance rapide qui donneraient des poulets vidés d'environ $500 \mathrm{~g}$ a été atteint. Considérant encore le fait que 66,4 p. 100 des poussins de l'effectif avaient un poids final situé entre 480 et $910 \mathrm{~g}$, les résultats obtenus dans ces conditions expérimentales semblent être satisfaisants.

En ce qui concerne les indices de consommation des rations de l'essai, aucune différence statistique n'a pu être relevée sur la base des valeurs absolues; ils se rangent dans l'ordre suivant : 2,72 pour $\mathrm{R}_{3} ; 2,81$ pour $\mathrm{R}_{2} ; 2,92$ pour $\mathbf{R}_{\mathbf{4}} ; 3,09$ pour $\mathbf{R}_{1}$ et 3,21 pour $\mathbf{R}_{\mathbf{t}}$.

Nous appuyant sur l'intervalle de 2,5-3 avancé par CALET (2), les indices obtenus avec les rations $R_{3}, R_{2}, R_{4}$ et $R_{1}$ sont accep- tables bien que pour cette dernière ration, lindice de 3,09 soit juste à la limite.

Sur la base des performances réalisées, la ration $R_{3}$ contenant 3 p. 100 de levure de brasserie séchée peut être retenue pour les rations de poulets d'engrais; une dose de 4 p. 100 dans la ration a causé une dépression de croissance.

\section{CONCLUSION}

L'essai qui précède a été mené avec des rations levurées à $1,2,3$ et 4 p. 100 et il en ressort :

1) que la levure de brasserie séchée a un effet positif dans la ration de poulet d'engrais, vu la supériorité des rations supplémentées par rapport à la ration témoin quant aux poids finals ;

2) que les indices de consommation s'amélioraient avec le taux croissant de levure pour les rations contenant 1,2 et 3 p. 100 de levure, le meilleur indice étant réalisé avec le taux de 3 p. 100 ;

3) que le taux de 3 p. 100 est souhaitable dans les rations de poulets d'engrais car il assure une meilleure croissance et un meilleur indice de consommation.

\section{SUMMARY}

Trial of dried brewers' yeast incorporation in diet for broiler

Four diets containing $1,2,3$ and 4 p. 100 yeast were tested and compared with a control diet. Feed conversion ratios were improving with the yeast increasing level for 1,2 and 3 p. 100 diets, the best ratio being obtained with 3 p. 100 level.

This last level also gave a best weight evolution while the 4 p. 100 level gave a weight rate decrease.

\section{RESUMEN}

Ensayo sobre la incorporación de levadura de cerveceria desecada en la ración de gallinas para carne

Se probaron y se compararon, con una ración testigo, cuatro raciones con 1 , 2,3 y 4 p. 100 de levadura.

Los indices de consumo iban mejorando con el aumento de la tasa de levadura en las raciones que contenian 1,2 et 3 p. 100 de ella; el mejor indice realizandose con la tasa de 3 p. 100.

Dicha tasa de 3 p. 100 permitió tambien obtener una mejora evolución del peso mientras que se observó una disminución de crecimiento con la tasa de 4 p. 100 . 


\section{BIBLIOGRAPHIE}

1. BRION (A.). Vade-Mecum du vétérinaire. Paris, Vigot, 1966.

2. CALET (C.). La production du poulet de chair, quelques facteurs importants. Rev. Elev., 1958, 23 : 7-10.

3. EWING (W. R.). Poultry Nutrition, 4. ed., New York, W. R. Ewing Publ., 1951.
4. MONGODIN (B.), RIVIERE (R.). Valeurs bromatologiques de 150 aliments de l'Ouest africain, Rev. Elev. Méd. vét. Pays trop., 1965, 18 (2) : 183-218.

5. WEGNER (R. M.). Zur Verwendung von Bierhefe, Biertreber/Bierhefe und Weizenkleie/Bierhefe im Kükenmastfutter, Dt. Geflügelwirtsch., 1970, 23 : 840-841. 